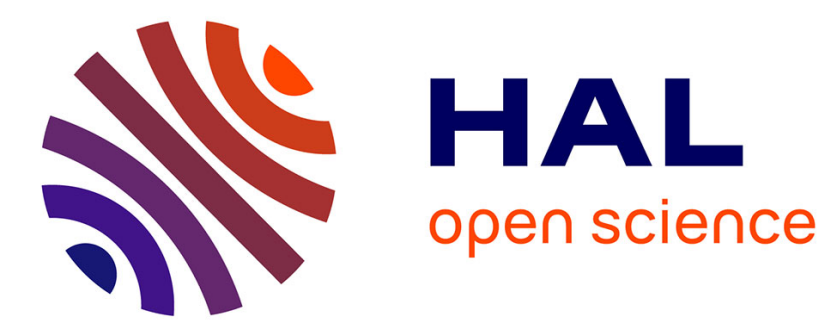

\title{
Guider les pas du spectateur numérique: la réintermédiation technique pensée à l'aune des valeurs historiques de la cinéphilie
}

Christel Taillibert

\section{To cite this version:}

Christel Taillibert. Guider les pas du spectateur numérique: la réintermédiation technique pensée à l'aune des valeurs historiques de la cinéphilie. Télévision, 2020. halshs-02938625

\section{HAL Id: halshs-02938625 \\ https://shs.hal.science/halshs-02938625}

Submitted on 15 Sep 2020

HAL is a multi-disciplinary open access archive for the deposit and dissemination of scientific research documents, whether they are published or not. The documents may come from teaching and research institutions in France or abroad, or from public or private research centers.
L'archive ouverte pluridisciplinaire $\mathbf{H A L}$, est destinée au dépôt et à la diffusion de documents scientifiques de niveau recherche, publiés ou non, émanant des établissements d'enseignement et de recherche français ou étrangers, des laboratoires publics ou privés. 


\title{
Guider les pas du spectateur numérique : la réintermédiation technique pensée à l'aune des valeurs historiques de la cinéphilie
}

\author{
Christel TAILLIBERT
}

Le développement sur les interfaces numériques des possibilités nouvelles que la délinéarisation des produits audiovisuels offrait aux spectateurs s'est rapidement accompagné d'un phénomène de réintermédiation technique, le plus souvent porté par des méthodes marketing agressives, sous la forme de pratiques de recommandation articulées à des logiques algorithmiques. La segmentation des publics ainsi pensée tend sans conteste à standardiser les décisions de consommation : alors que le (télé)spectateur entretient la sensation de devenir son propre programmateur, de bénéficier de «toujours plus d'individualisation, de mobilité et de flexibilité » (Jacquinot 2016), il s'enferme en réalité dans des cheminements d'individuation l'appelant à renouveler sans cesse les mêmes typologies d'expériences, ses désirs de consommation se voyant conditionnés par des propositions marketing normalisantes.

Ce «devenir grégaire des comportements» (Stiegler $2004: 24)$ que favorisent ces nouveaux environnements audiovisuels numériques ne peut satisfaire les acteurs qui abordent la fréquentation des œuvres cinématographiques et audiovisuelles à l'aune du concept de cinéphilie, concept que nous envisageons au prisme d'un rapport singulier aux œuvres, dont la création est appréhendée en tant que démarche artistique, et la découverte en tant que démarche culturelle, dans le contexte d'une opposition revendiquée, militante, aux fondements strictement commerciaux sur la base desquels s'est historiquement construite l'industrie cinématographique mondiale. La médiation cinéphile, telle qu'elle continue d'être valorisée en présentiel (festivals, cinémathèques, salles Art et Essai, etc.), met en avant l'idée d'un accompagnement du spectateur, d'un encadrement de son expérience cinématographique dans une matrice indérogeable fondée, entre autres, sur trois logiques que nous interrogerons tour à tour: une logique de prescription par laquelle le spectateur est guidé vers des œuvres 
sélectionnées en vertu de leurs qualités intrinsèques ; une logique de valorisation du partage de l'expérience, par laquelle la simultanéité de l'expérience permise par le caractère collectif de la projection est perçue comme le moteur d'une énergie éducative collective, misant sur «le devenir d'un public participant » (Ethis 2007 : 15) pour fonder l'acte de médiation ; et enfin un accompagnement éducatif, proposé par un « animateur », un «médiateur », qui tend à inscrire la découverte filmique dans une démarche d'appropriation culturelle individuelle et collective.

Comment réintroduire ces principes fondateurs dans le cadre sociotechnique aujourd'hui imposé par la Vidéo à la Demande (VàD) ? Comment adapter ces nouvelles logiques de visionnement aux valeurs fondatrices des acteurs cinéphiles français, valeurs qui sous-tendent un large écosystème incluant des acteurs institutionnels, économiques et associatifs ? Nous poserons l'hypothèse selon laquelle, tout en conservant les principes et l'ergonomie propre à l'univers de la VàD, les acteurs cinéphiles parviennent à réintroduire dans cet objet sociotechnique un «script» (Akrich 1990 : 2010) d'emploi cinéphile par lequel ils cherchent à « guider les pas » des usagers des plateformes numériques de mise à disposition de produits cinématographiques et audiovisuels, sans remettre en question le cadre éthique fondateur de la cinéphilie. La mise en avant de ces principes de réintermédiation dans la prise en charge du parcours de découverte de l'usager constituera ainsi l'axe problématique central de cet article.

Nous nous appuierons pour ce faire sur la littérature scientifique relative à l'économie et à la socio-économie des industries culturelles, à la sociologie des techniques, à l'économie de l'attention, ou encore à la cinéphilie dans ses acceptions traditionnelles comme renouvelées. Parallèlement, notre réflexion se développera sur la base d'une enquête de terrain vouée à l'analyse d'un corpus de quinze plateformes ${ }^{1}$, couplée à une série d'entretiens menés avec les créateurs/animateurs des plateformes ainsi retenues (les dates desdits entretiens seront précisées lorsque nous y aurons recours).

Nous développerons notre propos au gré de trois parties, qui aborderont successivement différentes dimensions de cette prise en charge du cheminement de l'usager par le dispositif sociotechnique de la VàD cinéphile. Dans une première partie, nous observerons la façon dont le cheminement de l'usager s'inscrit dans une cartographie particulière du site propre à traduire la dimension qualitative des œuvres qui lui sont proposées ; nous évoquerons dans une

\footnotetext{
${ }^{1}$ Ces plateformes sont les suivantes : Boutique ARTE; Benshi ; Bref Cinéma ; Bretagne \& Diversité ; Upopi ; Mémoire ; Ciel ; Le CiNéMa Club ; La Cinetek ; Le Kinetoscope ; KuB ; My French Film Festival ; Nowave ; Tënk ; La Toile ; UniversCiné.
} 
deuxième partie la façon dont sont réintroduites dans cet univers numérique des logiques de rendez-vous, destinées à construire un calendrier de visionnage pour les usagers, condition indispensable à un partage d'expériences dont nous évaluerons la possible déclinaison en ligne ; nous nous intéresserons enfin, dans une troisième et ultime partie, aux logiques d'accompagnement éducatif qui complètent ce script global.

\section{Une cartographie singulière de la VàD : logiques de prescription à l'œuvre}

Le premier écueil qu'ont cherché à contourner les acteurs cinéphiles de la VàD désireux de guider les usagers vers des œuvres retenues en vertu de critères qualitatifs était induit par les logiques de catalogue qui ont accompagné la délinéarisation des contenus et leur commercialisation sur Internet. Les agrégateurs de contenus et les grands groupes audiovisuels investissant ce marché se sont en effet lancés dans une « course au catalogue », dont l'ampleur affichée constituait un élément marketing fort à destination des usagers, et cela sans souci particulier de sélectivité : on promettait à ces derniers des possibilités de choix démultipliées, en vertu de l'utopie de l'accessibilité qui a accompagné le développement du numérique. Les logiques cinéphiles de prescription, basées sur une forte sélectivité et une attention poussée à la qualité des œuvres, ne pouvaient se satisfaire de cette immersion des usagers dans un ensemble de titres au sein desquels ils n'avaient la plupart du temps pas les moyens de séparer le bon grain de l'ivraie. Différentes stratégies ont donc été expérimentées pour réintroduire les valeurs constitutives de la prescription dans l'univers de la VàD, tout en l'inscrivant dans une tradition de la « qualité cinématographique » (Montebello 2003 ; Vernet 2017).

Le « catalogue sélectionné » et les logiques cartographiques cinéphiles

Quelques acteurs cinéphiles n'ont pas renoncé aux logiques de catalogue, mais ont fait le choix d'en renverser le principe consistant à évaluer la richesse de la proposition sur la base de critères quantitatif (le nombre de titres collationnés) pour adopter des critères strictement qualitatifs ${ }^{2}$. Ces « catalogues sélectionnés » assurent ainsi l'usager de naviguer dans une offre globale dont chacun des titres aura été retenu en vertu de ses qualités, de son intérêt cinéphilique. Ce choix est généralement vérifié lorsque l'acteur considéré peut se prévaloir d'une image de marque

\footnotetext{
${ }^{2}$ Les plateformes concernées dans notre corpus sont les suivantes : Boutique ARTE; Bretagne \& Diversité ; Mémoire ; La Cinetek ; Le Kinetoscope ; My French Film Festival ; UniversCiné.
} 
d'ores et déjà fortement reconnue sur le terrain de la qualité cinématographique (c'est le cas, par exemple, des déclinaisons numériques d'ARTE), ou lorsque qu'il s'appuie sur un écosystème à haute valeur culturelle qui lui permet de communiquer auprès des usagers quant aux qualités artistiques des productions rassemblées (ce que travaille à titre d'exemple la plateforme UniversCiné, mettant en avant le fait que son offre est issue de l'agrégation des catalogues des producteurs et distributeurs français indépendants - l'indépendance constituant une qualité fortement mise en avant dans le système de valeurs fondateur de la cinéphilie). Dans les deux cas, la stratégie de différenciation affichée par ces acteurs passe par un adossement à des organismes à forte valeur ajoutée d'un point de vue culturel, qui garantissent auprès des usagers la qualité du catalogue qui lui est proposé, la sélectivité qui a prévalu au choix des titres le composant.

Cette sélectivité affichée s'accompagne d'une forte volonté d'éditorialisation de la plateforme, par la création d'une cartographie grâce à laquelle l'usager sera appelé à se déplacer au sein du site, tout en mobilisant des critères de choix, de sélection, d'élection propres à la cinéphilie. Il s'agit en substance, pour les créateurs de ces sites, de proposer un parcours de navigation qui soit symboliquement chargé des valeurs cinéphiles. On peut ainsi relever, parmi ces marqueurs éditoriaux, la volonté de proposer une approche diachronique de la création cinématographique, fidèle à l'idée d'une culture cinématographique consciente des apports du passé, des influences que les œuvres, les courants, les mouvements exercent les uns sur les autres. Cette approche est, logiquement, tout particulièrement mise en avant sur une plateforme comme la Cinetek, dont la ligne éditoriale est construite sur la mise en avant du cinéma de patrimoine, ou sur Mémoire, directement vouée à des questions mémorielles. Mais on la retrouve aussi sur des plateformes plus traditionnelles, comme ARTE, Bref Cinéma, Benshi, etc. : les films «cultes », les «classiques »y sont en effet distingués comme des éléments incontournables de la constitution d'une culture cinéphile. Des outils de navigation comme des «lignes du temps» permettent ainsi à l'usager, sur certaines plateformes (Tënk, UniversCiné...), de bénéficier d'une visualisation de l'historisation des collections.

Parallèlement, l'approche géographique, propre à rendre compte de la richesse des collections au regard du critère de la diversité culturelle, constitue un autre choix d'aiguillage - clairement politique - prisé par les créateurs de ces plateformes. Il s'agit ainsi, comme l'exprimait Caroline Troin (entretien du 30 octobre 2017) de Bretagne \& Diversité, de mettre en lumière «des régions du monde où il y [a] des formes filmographiques peu diffusées », et d'engager l'usager sur la voie de la découverte de la multiplicité des cultures du monde et des 
productions cinématographiques dont elles sont à l'origine. Cet enjeu, fondateur de tout un pan de la cinéphilie, transparaît fortement dans les plateformes UniversCiné, Bretagne \& Diversité, Tënk (rubrique «Docmonde - Vues d'ailleurs »), et correspond à une orientation programmatique forte pour la Boutique ARTE, sans y être décliné sous forme d'aiguillages spécifiques. Il sera logiquement absent des plateformes essentiellement ou totalement éditorialisées autour du cinéma français (Mémoire, KuB, My French Film Festival, Bref Cinéma...).

Notons aussi la mise en place de marqueurs liés à une approche clairement a-normative, par laquelle vont être mises en valeur des productions distinguées par leur caractère atypique. Ce choix intervient encore une fois en parfaite continuité avec la politique poursuivie par les dispositifs traditionnels de médiation cinéphile qui ont toujours valorisé la programmation d'œuvres différentes - en termes de forme, de format, de support, de genre, de contenu -, au regard de ce qui était communément présenté dans les circuits classiques de diffusion des œuvres cinématographiques. Ce besoin relevant d'une stratégie de différenciation inhérente au système cinéphile se traduit sur les plateformes considérées par des fléchages spécifiques, qui tendent à conduire les usagers vers des films de court ou moyen métrage, vers des documentaires, des films d'avant-garde, expérimentaux, etc., donc toutes ces typologies de films écartés des circuits classiques de diffusion. Gilles Freissinier (entretien du $1^{\text {er }}$ juin 2017), d'ARTE, rendait ainsi compte de ce souci commun à l'ensemble de ces acteurs de la VàD cinéphile : «Éditorialement, le numérique permet aussi de concrétiser la vocation d'ARTE qui est de proposer l'accès à la culture ou à la créativité, la découverte de nouveaux talents, via des formes originales, une façon de nous raconter des histoires, ou de raconter des contenus originaux. » Notons par ailleurs que certaines plateformes de notre corpus ont inscrit dans leur ligne éditoriale même cette volonté de mettre en avant ces typologies de production atypique, en se spécialisant sur ces créneaux singuliers: le court métrage pour Bref Cinéma, le documentaire pour Tënk, le film anthropologique pour Bretagne \& Diversité, le film amateur pour Mémoire, le film de patrimoine pour la Cinetek... Le principe d'aiguillage est alors inscrit dans l'adéquation même avec la ligne éditoriale de la plateforme.

L'aiguillage par catégories de public est de la même façon privilégié, afin d'aider les parents à choisir des films adaptés à l'âge de leurs enfants. Ce souci répond au besoin fort de former les jeunes publics qui accompagne 1'histoire cinéphile, et que traduisent en particulier les programmes nationaux d'éducation à l'image promus par l'État français depuis les années 1980. Enfin, lorsque des marqueurs plus classiques sont adoptés, comme celui du genre que 
l'on peut retrouver dans la plupart des plateformes « strictement commerciales » de VàD, ils se voient déclinés par les acteurs cinéphiles au gré de subdivisions beaucoup plus riches et variées que ce qu'offrent les acteurs intégrés (UniversCiné, à titre d'exemple, ne propose pas moins de 21 entrées dans son onglet consacré à l'approche par genres).

Au-delà de ces principes généraux, la volonté de structurer le parcours de découverte de l'usager se traduit aussi par le recours à la pédagogie du ricochet: sur la base de la découverte d'un film, l'internaute va être invité à en découvrir un autre, peut-être moins facile d'accès. Loin de correspondre aux logiques d'étoilement développées sur les sites classiques, et qui encouragent à la reconduction à l'infini des expériences vécues, il s'agit ici de partir du désir de l'internaute, peut-être en lui proposant un film plus immédiatement attractif, pour l'emmener sur les chemins de la découverte cinéphile. Gilles Freissinier, d'ARTE, résume cette inclinaison lorsqu'il déclare : « Notre objectif, c'est toujours de montrer la diversité de notre offre, et de permettre à l'internaute qui est venu pour découvrir un programme qu'il connaissait d'en découvrir des nouveaux. » Il s'agit ainsi, par la confrontation à des œuvres diversifiées, par l'étonnement et l'intérêt qu'elles peuvent susciter, de mettre en place les conditions d'une formation, en autonomie, d'une culture cinéphile individuelle et partagée toujours plus exigeante.

Ainsi, de façon globale, il est intéressant de constater comment, au sein des catalogues sélectionnés propres à la VàD cinéphile, des aiguillages spécifiques sont réinvestis de sorte à accompagner l'usager dans ses déambulations au sein des collections, au gré de cheminements respectueux des valeurs, des objectifs, des moyens d'action propres au système cinéphile.

La « sélection tournante » : concentrer la fréquentation sur des chemins balisés

L'autre stratégie qui va caractériser le travail de prescription opéré par les acteurs cinéphiles se traduit, à l'inverse, par une remise en question des logiques de catalogue. Elle les conduit à interroger les conséquences de ces logiques de mise à disposition inhérentes au paradigme Pull (Benghozi ; Paris 2003) qui les sous-tend, et par lesquelles l'usager, face à une offre démultipliée, se retrouve démuni, confronté à des listings infinis de titres inconnus, et se voit incapable de prendre une décision de consommation. Les acteurs intégrés ont rapidement pris en considération l'anxiété que générait cette situation d'hyperchoix (Schwartz 2004), en y opposant rapidement des principes de réintermédiation mettant en avant les nouveautés d'une part (en termes de dates de production comme en termes d'entrée dans le catalogue) et les films 
les plus prisés par les internautes d'autre part - préceptes dont l'application concourut rapidement, en favorisant une «économie du hit» (Chantepie; Le Diberder 2010: 47), à contredire le modèle de la longue traîne proposé par Chris Anderson (2009).

Certains acteurs cinéphiles de la VàD ont ainsi pris conscience de l'impasse que pouvaient constituer les logiques de catalogue dans le cadre d'un travail de médiation, à l'image de Marie-Louise Khonji (entretien du 7 juillet 2017), créatrice du CiNéMa Club, qui déclarait :

«J'ai beaucoup observé comment la distribution évoluait au sein du numérique, et en regardant toutes les plateformes qui s'étaient créées, ce qui m'attristait un peu c'était la façon dont les films étaient présentés : c'était une jaquette, un poster parmi des milliers... On avait l'impression d'être dans un supermarché de films, on perdait un peu la magie de la salle de cinéma - qui fait partie de l'amour qu'ont les cinéphiles, du plaisir que quelqu'un a à découvrir un film. »

Aussi certains imaginèrent-ils d'autres principes pour fonder leur action en ligne, mettant en avant la nécessité de délester la proposition, en limitant, drastiquement, le nombre de titres disponibles. Cette offre volontairement limitée se calquait ouvertement sur les modèles prescriptifs inhérents au principe de programmation, comme le rappelait Joséphine Létang (entretien du 9 mai 2017), créatrice de la plateforme La Toile : «Quand on est cinéphile, mais pas forcément formé à ça, on se retrouve devant des millions de films sans savoir exactement ce que l'on veut regarder. Alors que la salle, en revanche, nous accompagne, fait une vraie sélection. Et l'idée, c'est de retrouver ce phénomène sur Internet. »

Pour mettre ce principe en pratique, certaines plateformes ont structuré leur proposition autour d'une sélection tournante, par laquelle seuls quelques titres étaient rendus disponibles, pendant un temps limité (c'est le cas en particulier des plateformes Ciel, Nowave, Le CiNéMa Club, Upopi, Bref Cinéma, Benshi, Tënk, La Toile, UniversCiné dans le cadre de son offre UnCut, ou encore La Cinetek dans la mise en avant de sa «Liste du mois »). En vertu de ce principe, le cheminement proposé à l'usager est maîtrisé, balisé, circonscrit, et complètement pensé par le «programmateur» : la fonction de prescription y est de fait considérablement valorisée. Parfois, le nombre de titres accessibles est tellement réduit (un pour Le CiNéMa Club, deux à quatre pour Nowave, 18 pour Ciel, 25 pour Upopi...) qu'ils sont présentés sur le même plan, dans un geste appelant l'usager, confiant dans les propositions prescriptrices de la plateforme, à les visionner tous, sans chercher plus avant à tracer des routes au sein de l'offre globale. Comme le revendiquait Marie-Louise Khonji, du CiNéMa Club, qui a poussé cette stratégie à l'extrême, «le fait qu'il y en ait qu'un seul [film] à la fois, c'est vraiment ce qui est prescripteur (...) Je dis aux usagers : “ n’y a que ça qui est recommandé cette semaine” ». Et 
même lorsque la proposition est quelque peu élargie, les programmateurs invitent en substance les usagers à monter dans un train qui les emmène de découverte en découverte, toutes les gares se proposant comme une halte opportune, sans que plus de discernement ne soit nécessaire de leur part. Pour Joséphine Létang (La Toile), il s'agit en substance de dire aux usagers : « Même si au premier abord, pour une raison ou pour une autre, vous ne seriez pas allés vers ça, nous on vous le conseille. » $\mathrm{Ce}$ « nous » ici mis en avant, qui est parfois un « je » selon l'organisation des plateformes considérées, réintroduit au premier plan l'idée d'une subjectivité dans les choix de programmation, revendiquée comme fondatrice de l'acte de médiation. L'adéquation de la proposition avec l'horizon d'attente cinéphile des usagers peut alors permettre la reconnaissance de la fonction de prescription exercée par les plateformes considérées, et l'établissement d'une relation de confiance avec la proposition culturelle garante d'un plein engagement des usagers sur le long terme.

\section{Des rendez-vous cinéphiles en ligne : construction d'un calendrier de visionnage partagé}

Le principe d'une sélection tournante, retenue par nombre d'acteurs cinéphiles, traduit par ailleurs une claire volonté de l'opposer au « Any Time » propre à la logique de l'ATAWAD. Cette configuration particulière constitue en effet un cadre permettant de travailler les régimes d'attention dans le contexte d'Internet où, comme le notait Françoise Benhamou (2014 : 49), « l'offre est infinie et l'attention devient une ressource rare ». À titre d'exemple, la logique festivalière adoptée par My French Film Festival (MFFF) - qui constitue une forme spécifique de sélection tournante - répond directement à ce besoin d'événementialiser la proposition, de capter l'attention pour fonder un travail efficace de médiation. Le créateur de cette plateforme, Jean-Rémi Ducourtioux (entretien du 7 juillet 2017), insistait sur ce point : «C'est vraiment important cette dimension événementielle, pour que le public l'attende ce festival, en tous cas attende ces films-là et réponde présent. Je pense que si on est présent toute l'année, on disperse l'intérêt. »

Convoquant les principes inhérents au marketing de la rareté, le principe de la sélection tournante mise par ailleurs sur la crainte que peuvent éprouver les usagers de voir disparaître la proposition de découverte qui leur est faite, ce qui constitue une incitation forte à visionner rapidement les films disponibles. Certaines plateformes usent même d'un compte à rebours pour mieux traduire l'urgence qu'il y a à profiter de la présence en ligne des titres proposés. Les marqueurs sémantiques propres au registre de la sélection tournante travaillent clairement cette idée d'une « saisonnalité » de la proposition, donc de son caractère périssable, éphémère 
(on y parle par exemple des «courts du moment» [Upopi], de la « sélection du moment » [Ciel], de la « programmation mensuelle »[La Toile], etc.)

Notons que d'autres stratégies que la sélection tournante travaillent la question de l'événementialisation, en particulier pour les plateformes qui recourent aux logiques de catalogue. Les animateurs de ces dernières cherchent en effet à créer une émulation autour de l'actualité de la plateforme, en mettant en avant les nouveaux titres qui s'agrègent au catalogue initial (principe marketing partagé par les acteurs classiques de la VàD), ou encore en développant un travail d'éditorialisation, la plupart du temps - sans que cela ne soit restrictif en interaction avec l'actualité politique, sociale ou culturelle. Ceci suppose des connaissances accrues du travail de programmation, et permet en même temps de réintroduire une temporalité dans l'offre de la plateforme. Comme le déclarait Cécile Horreau (entretien du 10 mai 2017) du Kinetoscope, «L'idée était de pouvoir faire des propositions, de faire vivre la plateforme, que ce ne soit pas un objet figé, qui ne vivrait que par ses nouveaux apports. » Par ailleurs, cette dernière tendance tend aussi à inscrire la découverte cinéphilique dans le cadre d'une pratique globale réfléchissant le cinéma comme un outil de compréhension du monde, au-delà des pures logiques de divertissement dans lesquelles on entend le confiner, et en parfaite continuité avec les principes fondateurs de l'action culturelle cinéphile. Les réseaux sociaux se voient largement investis pour accompagner ce travail d'événementialisation, comme le rappelait Gilles Freissinier (ARTE) :

« On peut aussi redonner de la vie, et ça se fait beaucoup en ce moment, à travers le direct sur les réseaux sociaux : ça devient un enjeu majeur parce que les réseaux sociaux se positionnent comme des télés bis, avec le direct, ça redonne de l'événement, car la mise en ligne d'un live sur Facebook est notifiée à l'ensemble des fans, et du coup, on a une façon très rapide de toucher, d'avertir un public sur le fait qu'il se passe quelque chose, qu'il y a un événement. On a différents leviers pour donner de l'urgence, de l'événement à nos contenus. »

Ainsi, les acteurs cinéphiles de la VàD travaillent tous les questions de temporalité, cherchent à recréer des moments forts, des calendriers de découverte, au-delà des logiques de mise à disposition qui écartent de fait l'idée d'une date et d'un horaire précis autour desquelles s'articule la rencontre programmée avec une œuvre cinématographique (comme il est de mise dans les salles, les festivals, les ciné-clubs, les cinémathèques, ou encore à la télévision). Il s'agit d'un levier d'action fondamental dans cette tentative d'accompagnement de l'usager dans un parcours de découverte en ligne dont les circonvolutions, comme le calendrier, auront été pensées en accord avec les besoins de la médiation cinéphile. 
La réintroduction d'une dimension temporelle à la proposition culturelle en ligne travaille directement le concept de « rendez-vous », élément central dans la structuration de la consommation culturelle ${ }^{3}$, et tout particulièrement dans le registre de la cinéphilie qui ne s'est jamais définie comme une pratique solitaire, mais au contraire comme une construction sociale collective. Le partage des expériences constitue dans ce contexte un élément fondateur de l'acte de médiation. Il s'agit donc, pour les animateurs de ces plateformes, de chercher à créer, tout en respectant le cadre de l'ATAWAD, un calendrier de partage des expériences susceptible de permettre une horizontalité des échanges entre des usagers qui auront globalement découvert les mêmes films au même moment, encore une fois sur le modèle historiquement proposé par la salle. Comme le rappelait Emmanuel Ethis (2007 : 18),

«Les sociabilités cinématographiques que la salle de cinéma a organisées en rationalisant le concept même de rendez-vous : un lieu, un horaire, un programme, une programmation, une rencontre, une sociabilité. Ce dernier point est cardinal, car c'est surtout parce que les films fournissent socialement la plus formidable des occasions de se connaître, d'éprouver son jugement de goût avec celui des autres, de reconnaître ceux qui aiment les mêmes choses que la sortie au cinéma demeure, et ce de très loin la pratique dominante en matière de sortie culturelle ».

La tentation de création d'espaces de sociabilité autour de ces plateformes, dans le parfait prolongement de ces principes structurants, s'avère toutefois assez peu probante à ce jour. Lorsque des espaces de commentaires sur les films sont proposés (ce qui est le cas sur ARTE, Mémoire, MFFF, UniversCiné et Benshi), ils se révèlent peu utilisés, et lorsque c'est le cas, donnent lieu à des jugements et recommandations généralement péremptoires et non argumentés, loin de l'exercice de l'expression d'une pensée critique valorisée par la cinéphilie. Les mêmes observations peuvent être développées lorsque cet espace d'expression autonome se déplace sur les réseaux sociaux associés à ces plateformes. L'absence d'un « organisateur humain du débat », comme il se manifeste dans les dispositifs de médiation en présentiel, explique probablement largement ce peu d'attrait que constitue l'invitation au partage d'expérience à laquelle certaines plateformes invitent leurs usagers.

\section{Les logiques d'accompagnement éducatif adaptées à la VàD}

\footnotetext{
${ }^{3}$ Laurent Fonnet $(2008$ : 148) rappelait par exemple qu’à la télévision, « avec la multiplication des chaînes, le téléspectateur est de plus en plus sensible à la notion de "rendez-vous" qui le rassure et est un point d'ancrage dans la grille de programmes ».
} 
Si les dispositifs d'éducation horizontale proposés par ces plateformes relèvent clairement de l'expérimentation, et cherchent encore les voies de leur pleine maturation, des volontés d'accompagnement de l'usager vont s'affirmer de façon beaucoup plus significative lorsque l'on s'attache aux logiques de médiation verticale. Ainsi, les «scripts d'emploi» proposés par ces dispositifs sociotechniques singuliers supposent, au-delà du fait de diriger, comme on l'a vu, vers des films différents et au gré de temporalités pensées en amont, de s'approprier différemment les œuvres cinématographiques. Jean-Rémi Ducourtioux, de MFFF, affirmait ainsi : «On espère en premier lieu être prescripteurs d'un cinéma déjà différent, et ensuite d'une façon de le consommer différente ». Cette «façon différente de consommer » tient avant tout aux accompagnements éducatifs qui vont être mis en œuvre par les acteurs de ces plateformes, destinés à créer des rituels médiationnels d'accompagnement qui vont structurer la rencontre entre l'horizon d'attente de l'usager cinéphile d'une part, qui construit sa fréquentation des œuvres dans une perspective culturelle, et les velléités éducatives des acteurs de la médiation d'autre part, qui vont chercher dans le cadre offert par le numérique les moyens d'adapter les schèmes d'action qui caractérisent traditionnellement la médiation cinéphile.

Cette structuration symbolique de l'espace de médiation va essentiellement se traduire par la proposition d'un étayage informationnel, destiné à aider l'usager à dépasser l'état premier de « sidération » (Meirieu 2004) occasionné par la projection cinématographique, en lui offrant la possibilité de confronter le fruit de son expérience à des registres d'analyse à la fois esthétiques, historiques, sociologiques, géopolitiques, etc. L'usager va ainsi être invité, dans son parcours de découverte, à consulter un certain nombre de documents mis à sa disposition, destinés à lui permettre de mieux comprendre le contexte de production et de réalisation de l'œuvre visionnée, ainsi que la place qu'elle occupe dans le vaste panorama de l'art cinématographique. Ces propositions constituent une plus-value pour les usagers, dans le cadre d'une démarche éducative propre à travailler leur propre compétence spectatorielle (Leveratto 2003) pensée par les créateurs de ces plateformes. Là encore, on observe une symétrie indéniable avec les pratiques courantes en présentiel, qui s'appuient largement sur le recours à différents supports d'accompagnement informationnels, sous forme orale (les « présentations » traditionnellement pratiquées avant la projection, ou les rencontres avec des invités qui lui font suite), sous forme écrite (fiches techniques, catalogues, monographies ou revues...), sous forme d'expositions, etc. 
La mise à disposition de documents écrits constitue la forme la plus répandue dans le cadre de cet étayage informationnel pratiqué par les plateformes cinéphiles, quand bien même de façon plus anecdotique d'autres typologies de documents sont aussi expérimentées. Il s'agit souvent, à travers des textes plus ou moins longs, de mettre en avant les réalisateurs dont les œuvres sont présentées, en conformité avec les préceptes propres à la politique des auteurs qui a accompagné la construction du système de valeurs cinéphile. L'ambition consiste donc à replacer la démarche du réalisateur au cœur de la réflexion, en concevant le geste de création comme élément premier de compréhension de l'œuvre d'art. On trouve ainsi proposés des encarts informatifs ou portraits plus complets qui présentent les metteurs en scène, mais aussi des interviews. Celles-ci sont parfois réalisées pour les besoins du site par ses animateurs, comme c'est le cas sur Bretagne \& Diversité qui en propose une retranscription écrite, ou sur UniversCiné et MFFF qui les offrent à la consultation sous la forme de vignettes vidéo. MFFF et Bref Cinéma bénéficient ponctuellement de captations de Master classes organisées réciproquement par Unifrance et la Plateforme du court métrage. Ces contenus exclusifs constituent des atouts indéniables dans le cadre de la stratégie de différenciation que poursuivent ces plateformes. Des logiques d'étoilement sont par ailleurs mises à profit pour travailler cette volonté d'étayage et guider l'usager vers des sources externes d'information, dans un geste qui replace la découverte du film dans une démarche culturelle globale. Les ressources nationales en matière «d'éducation à l'image » (Transmettre le cinéma, Nanouk, L'Enfant et le $7^{\mathrm{e}}$ Art, Ecole et cinéma, l'AFCAE...) constituent ainsi, en particulier pour les plus jeunes internautes, des lieux de prédilection vers lesquels des liens les redirigent, traduisant cette solidarité constitutive du système cinéphile au niveau national.

Les documents d'accompagnement qui sont proposés s'écartent parfois de cette première direction auteuriste pour amener l'usager à réfléchir et comprendre les thématiques que soulèvent les films qui lui sont proposés : le geste éducatif se déplace alors de l'éducation à l'image cinématographique vers l'éducation par l'image cinématographique, en conformité avec la multiplicité des approches que recouvre en réalité le vaste territoire de la médiation cinéphile. Car comme le rappelait Xavier Lardoux (2014 : 17), «Le cinéma, en tant qu'art, amène naturellement à se pencher sur de grands sujets, qu'ils soient métaphysiques, existentiels ou historiques ». Les films proposés sont donc envisagés sous la double facette d'objets artistiques et d'objets sensibles ouverts sur le monde, conformément à la multiplicité des investissements éducatifs dont a été historiquement l'objet le cinéma, en particulier de la part des mouvements d'éducation populaire, et que l'on retrouve dans le discours de tous les acteurs 
cinéphiles aujourd'hui. Gilles Freissinier (entretien du $1^{\text {er }}$ juin 2017) insistait ainsi sur le fait qu'ARTE entendait donner à voir des « programmes qui doivent nous permettre de mieux décrypter le monde dans lequel on vit, et donner accès à cette culture, que ce soit par la fiction, par le documentaire ou autre ». La plateforme Bretagne \& Diversité a tout particulièrement travaillé cette dimension, et propose des ressources très diversifiées (fiches informatives, bibliographies, contributions d'universitaires ou journalistiques, ...). Cette volonté de lier la découverte filmique à une découverte du monde est très présente dans les éléments de communication et d'éditorialisation qui accompagne la mise à disposition des films par les acteurs de ces plateformes, au-delà même de la présence de documents d'accompagnement spécifiques qui concerne une minorité d'acteurs (Bretagne \& Diversité, My French Film Festival, KuB). Elle se traduit par exemple par l'articulation de l'offre autour de thématiques (ARTE, UniversCiné, Benshi, Tënk, Le Kinetoscope, Bretagne \& Diversité, KuB, Mémoire, My French Film Festival) : ces regroupements de films permettent à l'usager de multiplier sur un sujet donné des visions du monde complémentaires, éventuellement divergentes, qui lui permettent d'approfondir sa réflexion personnelle sur la question retenue.

Au-delà de cette volonté, partagée par l'ensemble des acteurs, de fournir un étayage informationnel, quelques plateformes (Le Kinetoscope, Benshi) expérimentent d'autres formes d'accompagnement éducatif, sous la forme de parcours thématiques destinés à embrasser, à travers la découverte de plusieurs films réunis à cet effet, différentes perspectives relatives à une question unique. Conformes à la proposition pédagogique que vantait Alain Bergala (2002 : 118) à travers l'idée de «fragments mis en rapport », ces parcours de découverte tendent à explorer, par la confrontation aux œuvres plus que par l'analyse, des « questions de cinéma ». C'est par le biais de cette expression en effet que la plateforme Le Kinetoscope, à titre d'exemple, décline la proposition. Sa démarche est ainsi expliquée sur le site :

«Explorez des grandes questions de cinéma à partir d'un corpus de films pour envisager sous différents angles un aspect spécifique du cinéma (esthétique, technique, scénaristique). Il s'agit d'offrir pour chacune de ces questions, non pas une illustration, mais un certain nombre de films y répondant avec des approches distinctes. Confronter les films entre eux, pointer les similarités pour relever les singularités permet alors de problématiser la thématique initiale et de faire émerger un questionnement ${ }^{4} . »$

\footnotetext{
4 Site Le Kinétoscope, onglet «Question de cinéma», consulté le 4 octobre 2019
} http://www.lekinetoscope.fr/questions-de-cinema 
Conformément au principe de la VàD, liée à la mise à disposition d'œuvres intégrales, ces parcours s'articulent autour de films choisis, mais sans que les questions de mise en scène visées ne soient isolées - comme cela est le cas par la mise en relation d'extraits choisis dans la proposition d'Alain Bergala. Mais il s'agit toutefois d'expérimentations intéressantes dès lors qu'elles offrent une nouvelle traduction de cette la volonté de guider les pas de l'usager dans une démarche clairement pédagogique d'apprentissage, ici en l'occurrence du langage cinématographique.

\section{Conclusion}

Nous nous sommes attachés, au cours de cet article, à mettre en évidence la façon dont les plateformes de vidéo à la demande qui inscrivent leur action dans une démarche cinéphile cherchent à accompagner l'usager dans son parcours de découverte filmique, à guider ses pas dans le processus de découverte de l'art cinématographique. Ce processus de réappropriation du dispositif de la VàD permet aux acteurs cinéphiles, attachés à l'idée d'une médiation, d'une triangulation de la proposition, de réintroduire une fonction de prescription, d'accompagnement, au-delà de la simple fonction d'intermédiation technique propre au dispositif. Ce phénomène est intéressant dès lors qu'il témoigne d'une remise en cause des médiations techniques telles qu'elles ont été pensées par les industries culturelles, uniquement en fonction des modèles d'affaires qui les sous-tendent. En réintroduisant des schèmes culturels d'orientation dans la proposition, des temporalités partagées dans la découverte cinématographique, ou encore des prolongements éducatifs qui modifient le rapport entretenu aux œuvres, les acteurs cinéphiles de la VàD entendent réintroduire de nouvelles attentes au cœur de l'utopie de l'accessibilité qui a accompagné le développement du numérique, en redonnant une place à ces intermédiaires traditionnels, ces «tiers représentant le pôle instituant » (Fichez 2003 : 111) sur lesquels se fonde le système cinéphile. Ils contribuent ainsi à repenser, dans le contexte du numérique, les processus d'individuation indispensables à ce système dans son ensemble, par lesquels les cinéphiles pourront bénéficier d'une offre numérique proposant, d'un point de vue du système de valeurs, une continuation de leurs pratiques culturelles en présentiel, tandis que des usagers non « convertis » à la cause pourront donner du sens à leur consommation culturelle grâce à l'introduction d'un cadre symbolique structurant. 


\section{Bibliographie}

AKRICH Madeleine (1990), « De la sociologie des techniques à une sociologie des usages : l'impossible intégration du magnétoscope dans les réseaux câblés de première génération », Techniques et culture, $\mathrm{n}^{\circ} 16$ [en ligne] mis en ligne le 24 juin 2006 https://halshs.archivesouvertes.fr/halshs-00081710/

AKRICH Madeleine (2010), « Comment décrire les objets techniques?», Techniques \& Culture, $\mathrm{n}^{\circ} 54-55$ [en ligne] Disponible sur Revue.org, mis en ligne le 30 janvier 2013, http://tc.revues.org/4999

Alava Séraphin et ETEVE Christiane (1999), « Médiation documentaire et éducation », Revue française de pédagogie, ${ }^{\circ} 127$, pp. 119-164 [en ligne] consulté le 15 octobre 2017 http://www.persee.fr/doc/rfp_0556-7807_1999_num_127_1_1090

ANDERSON Chris (2009), La longue traîne : la nouvelle économie est là !, Paris, PearsonVillage mondial.

BENGHOZI Pierre-Jean et PARIS Thomas (2003), « De l'intermédiation à la prescription : le cas de la télévision », Revue française de gestion, $\mathrm{n}^{\circ} 142$, pp. 205-227.

BenHAMOU Françoise (2014), « La rareté et les flux numériques. La valeur de l'attention », Esprit, pp. 44-53.

Bergala Alain (2002), L'hypothèse cinéma - Petit traité de transmission du cinéma à l'école et ailleurs, Paris, Cahiers du cinéma, coll. Essais.

ChAntePIE Philippe et Le Diberder Alain (2010), Révolution numérique et industries culturelles, Paris, la Découverte.

CRETOn Laurent (1997), Cinéma et marché, Paris, Armand Colin.

ÉTHIS Emmanuel (2007), «Le cinéma, cet art subtil du rendez-vous », Communication et langages, L'énonciation éditoriale en question, ${ }^{\circ} 154$, pp. 11-21.

FISCHER Elisabeth (2003), «La médiation au risque de l'industrialisation », Notions en Questions, ${ }^{\circ} 7$, Médiation, médiatisation et apprentissages.

FONNET Laurent (2010), La programmation de la télévision à l'ère numérique. Comment ça marche?, Paris, Dixit.

JACQUINOT-DELAUNAY Geneviève (2011), « De l'éducation aux médias aux médiacultures : faire évoluer théories et pratiques », Qu'enseigne l'image? Qu'enseigner par l'image? [en 
ligne] e-dossier de l'audiovisuel mis en ligne en janvier 2011 http://www.ina-expert.com/edossier-de-1-audiovisuel-qu-enseigne-1-image-qu-enseigner-par-l-image/de-l-education-aux$\underline{\text { medias-aux-mediacultures-faire-evoluer-theories-et-pratiques.html }}$

LARDOUX Xavier (2014), Pour une politique européenne d'éducation au cinéma, Paris, Centre national de la cinématographie et de l'image animée.

LeVeratto Jean-Marc (2003), « Histoire du cinéma et expertise culturelle », Politix, nº61, pp. $17-50$.

MEIRIEU Philippe (2004), «Images, de la sidération à l'éducation », Conférence donnée au colloque "Enfants de cinéma", octobre 2004 [en ligne] https://www.meirieu.com/ARTICLES/IMAGES.pdf

MonTEBello Fabrice (2003), «Des films muets aux films parlants. Naissance de la qualité cinématographique », Politix, ${ }^{\circ} 61$, pp. 51-80.

RoJAS Estrella (2007), «Internet, outil de médiation culturelle. Vers un modèle de spécification basé sur les figures de médiation » [en ligne] Actes du colloque «Collaborer, échanger, inventer - Expériences de réseaux » (Hammamet, 29-31 octobre 2007). http://ticri.univ-lorraine.fr/ticrih2ptm.fr/index.php/H2PTM_(2007)_Rojas

SCHWARTZ Barry (2004), The Paradox of Choice: Why More is Less. New York, HarperCollins. STIEGLER Bernard (2004), «Le désir asphyxié, ou comment l'industrie culturelle détruit l'individu », Le monde diplomatique, $\mathrm{n}^{\circ} 6$, pp. 24-25.

VERNET Guillaume (2017), Aux origines d'un discours critique : la «tradition de la qualité » et la « qualité française ». La bataille de la qualité ou la mise en place du soutien de l'État aux films de qualité en France (1944-1953), Thèse de Doctorat, Université Rennes II. 